\title{
DETERMINAÇÃO DA EVAPOTRANSPIRAÇÃO MÁXIMA E REAL E DO FATOR DE SENSIBILIDADE AO DÉFICIT HÍDRICO DA MELANCIA EM CANINDÉ, CE
}

\author{
Alexandre Aires de Freitas ${ }^{1}$, Francisco Marcus Lima Bezerra ${ }^{2}$ \\ \& Francisco das Chagas Barros Fontenele ${ }^{3}$
}

\begin{abstract}
RESUMO
Este trabalho foi desenvolvido sob condições de campo e teve como objetivo determinar a evapotranspiração máxima $\left(\mathrm{ET}_{\mathrm{m}}\right)$ e real $\left(\mathrm{ET}_{\mathrm{r}}\right)$ e o fator de sensibilidade da cultura $\left(\mathrm{k}_{\mathrm{y}}\right)$ da melancia (Citrullus lanatus Thumb. Mansf.) cultivar "Crimson Sweet"; para tal, foi conduzido um experimento no município de Canindé, CE. Utilizaram-se oito tratamentos com quatro repetições cada um, adotando-se o delineamento experimental de blocos ao acaso. Os tratamentos foram definidos em função da época de aplicação do déficit hídrico e o ky foi obtido através da relação entre a queda de rendimento relativo $\left(1-\mathrm{y}_{\mathrm{r}} / \mathrm{y}_{\mathrm{m}}\right)$ e o déficit de evapotranspiração relativa $\left(1-\mathrm{ET}_{\mathrm{r}} / \mathrm{ET}_{\mathrm{m}}\right)$. As evapotranspirações máxima e real da melancia foram calculadas pelo método do balanço hídrico em volume de solo controlado em campo, que se apresentou confiável na determinação do consumo de água pela cultura. A sensibilidade ao déficit hídrico $\left(\mathrm{k}_{\mathrm{y}}\right)$ em cada estádio variou na seguinte ordem decrescente: floração, desenvolvimento dos frutos e vegetativo.
\end{abstract}

Palavras-chave: Citrullus lanatus, balanço hídrico, evapotranspiração

\section{DETERMINATION OF MAXIMUM AND ACTUALEVAPOTRANSPIRATION AND WATER DEFICIT SENSIBILITY FACTOR IN WATERMELON AT CANINDÉ, CE}

\begin{abstract}
This research was performed under field conditions and its main purpose was to determine the maximum $\left(\mathrm{ET}_{\mathrm{m}}\right)$ and the atual evapotranspiration $\left(\mathrm{ET}_{\mathrm{r}}\right)$ and establish the sensibility factor $\left(\mathrm{k}_{\mathrm{y}}\right)$ to the water deficit of the watermelon (Citrillus lanatus Thumb. Mansf.) cultivar "Crimson Sweet". The experiment was conducted in the district of Canindé, CE. Eight treatments with four replications were used, adopting a randomized block design. The treatments were defined according to the time of water deficit induction and $\mathrm{k}_{\mathrm{y}}$ was obtained through the relation between the reduction of relative efficiency $\left(1-\mathrm{y}_{\mathrm{r}} / \mathrm{y}_{\mathrm{m}}\right)$ and the deficit of relative evapotranspiration $\left(1-\mathrm{ET}_{\mathrm{r}} / \mathrm{ET}_{\mathrm{m}}\right)$. The maximum and actual evapotranspiration of watermelon were calculated by the method of water balance in a controlled soil volume, in a field which appeared reliable for the determination of the crop water consumption. The $\mathrm{k}_{\mathrm{y}}$ varied in each period, in the following decreasing order: flowering, fruit development and vegetative.
\end{abstract}

Key words: Citrullus lanatus, water balance, evapotranspiration

\footnotetext{
${ }^{1}$ Eng. Agrônomo, M.Sc. em Agronomia, R. Ten. Jaime Andrade 261, Aerolândia, CEP 60850 - 720, Fortaleza, CE. Fone: (085) 227 0121, E-mail: foras00t@zaz.com.br

${ }^{2}$ Prof. Dr. Departamento de Engenharia Agrícola, DENA/UFC, Fone: (085) 288 9763, CP 12.168, CEP 60450 - 760, Fortaleza, CE

${ }^{3}$ Eng. Agrônomo, SDR-CE. Fone: (085) 225 5292, Fortaleza, CE
} 


\section{INTRODUÇÃO}

O Brasil apresentou aumento de $10 \%$ em sua área plantada com melancia, no biênio 1994-1995, atingindo 79.683 ha de área plantada em 1995, ano em que a produção nacional chegou a 254.412.000 de frutos. O maior Estado produtor foi Pernambuco, seguido do Rio Grande do Sul e Bahia e o Ceará aparece na $17^{\text {a }}$ posição, com uma produção anual de 923.000 frutos (Anuário Estatístico do Brasil, 1996).

Nas áreas irrigadas do Ceará, o cultivo de melancia vem crescendo consideravelmente nos últimos anos, principalmente por alcançar bons preços, tanto no mercado interno como no externo, atingindo um aumento de $5 \%$ em sua área plantada no biênio 1994-1995 (Anuário Estatístico do Ceará, 1995).

A evapotranspiração máxima $\left(\mathrm{ET}_{\mathrm{m}}\right)$ refere-se às condições em que a água é suficiente para o crescimento e desenvolvimento de uma cultura sadia, sem restrição, cultivada em grandes áreas sob condições ótimas de manejo agronômico e de irrigação (Doorenbos \& Kassam, 1994).

Reichardt (1990) define a evapotranspiração máxima $\left(\mathrm{ET}_{\mathrm{m}}\right)$ como a máxima perda de água que certa cultura sofre em dado estádio de desenvolvimento, quando não existe restrição de água no solo. Ele afirma, também, que a evapotranspiração real $\left(\mathrm{ET}_{\mathrm{r}}\right)$, é a que de fato ocorre. Se houver água disponível no solo e o fluxo de água na planta atender à demanda atmosférica, $\mathrm{a} \mathrm{ET}_{\mathrm{r}}$ será igual a $\mathrm{ET}_{\mathrm{m}}$; se houver restrição de água no solo e a demanda atmosférica não for atendida, a $\mathrm{ET}_{\mathrm{r}}$ será menor que a $\mathrm{ET}_{\mathrm{m}}$. De acordo, ainda, com o autor, a disponibilidade de água afeta a produtividade e a situação ideal para a cultura seria a $\mathrm{ET}_{\mathrm{r}}$ igual a $\mathrm{ET}_{\mathrm{m}}$. Toda vez que a $\mathrm{ET}_{\mathrm{r}}<\mathrm{ET}_{\mathrm{m}}$, existe restrição de água e a produtividade pode ser afetada, razão pela qual a $\mathrm{ET}_{\mathrm{m}}$ é utilizada para se calcular a demanda climática máxima de uma cultura, em projetos de irrigação.

A irrigação, quando não é bem conduzida na cultura da melancia, pode causar danos indesejáveis, como salinização do solo, baixa produtividade e qualidade irregular dos frutos. Segundo Doorenbos \& Kassam (1994) a resposta da cultura em rendimento produtivo, ao suprimento de água, é quantificada através do fator de resposta da cultura $\left(\mathrm{k}_{\mathrm{y}}\right)$ que relaciona a queda de rendimento relativo $\left(1-\mathrm{y}_{\mathrm{r}} / \mathrm{y}_{\mathrm{m}}\right)$ com o déficit de evapotranspiração relativa $\left(1-\mathrm{ET}_{\mathrm{r}} / \mathrm{ET}_{\mathrm{m}}\right)$. Os autores concluíram que a relação entre o rendimento relativo $\left(\mathrm{y}_{\mathrm{r}} / \mathrm{y}_{\mathrm{m}}\right)$ e a evapotranspiração relativa $\left(\mathrm{ET}_{\mathrm{r}} / \mathrm{ET}_{\mathrm{m}}\right)$ é linear e válida para déficits hídricos até cerca de 50\%. Afirmam, ainda, que em condições de suprimento de água limitado a cultura sofrerá maior perda de rendimento quanto maior for o valor de $\mathrm{k}_{\mathrm{y}}$. Este fator apresentase, portanto, como base para o manejo racional de água em relação à produção irrigada.

$\mathrm{Na}$ tentativa de fornecer aos produtores informações a respeito da exploração irrigada dessa cultura foi instalado, no período de outubro de 1997 a janeiro de 1998, no município de Canindé, um experimento sob condições de campo, com o objetivo de determinar a evapotranspiração máxima e real e o fator de resposta ao déficit hídrico da melancia (Citrullus lanatus) cultivar "Crimson Sweet".

\section{MATERIAL E MÉTODOS}

O experimento foi conduzido no período de 26 de outubro de 1997 a 6 de janeiro de 1998, no Sítio Recanto, município de Canindé, CE, situado nas coordenadas geográficas de $4^{\circ} 21^{\prime} 32^{\prime \prime}$ latitude Sul e longitude $39^{\circ} 18^{\prime} 42^{\prime \prime}$, a oeste de Greenwich e altitude de 149,73 m; a precipitação normal anual da região é de $756,10 \mathrm{~mm}$ e o clima, semi-árido e com chuvas irregulares, é classificado, segundo Köppen como do tipo BSh (Vianello \& Alves, 1991). Os dados climáticos referentes ao período de execução do ensaio foram obtidos através de um posto de coleta de dados meteorológicos instalado no local do experimento, equipado com anemômetro, termohigrógrafo, pluviômetro e tanque "classe A".

O solo foi caracterizado através de análises físicas, no Laboratório de Hidráulica do Departamento de Engenharia Agrícola, e químicas, realizadas no Laboratório de Análises de Solo do Departamento de Solos da Universidade Federal do Ceará. As curvas características de água no solo, nas profundidades de $0,10,0,30,0,50$ e $0,70 \mathrm{~m}$, foram obtidas no Laboratório de Física do Solo do Departamento de Engenharia Agrícola da UFC, utilizando-se amostras indeformadas e acondicionadas em cilindros do tipo Uhland e sua coleta foi feita em uma trincheira aberta na área experimental. As amostras foram submetidas às tensões de $0,2,4,6,8,28,50,100,300$ e 700 $\mathrm{kPa}$ em funil de Haines, e em câmara de pressão de Richards. De posse dos valores de umidade da base de volume $\left(\mathrm{q}, \mathrm{m}^{3} \mathrm{~m}^{-3}\right) \mathrm{e}$ potencial mátrico de água no solo ( $\left.\mathrm{y}_{\mathrm{m}}, \mathrm{cm} . c . \mathrm{a}\right)$ obtiveram-se os parâmetros a, $n$ e $m$ da equação de van Genuchten, através do programa computacional Curvaret, descrito por Dourado Neto et al. (1990). A expressão matemática do modelo de Genuchten que faz a transformação do potencial matricial lido nos tensiômetros em umidade volumétrica, está expressa a seguir:

$$
\theta_{\mathrm{a}}=\theta_{\mathrm{r}}+\frac{\theta_{\mathrm{s}}-\theta_{\mathrm{r}}}{\left[1+\left(\alpha\left|\psi_{\mathrm{m}}\right|\right)^{\mathrm{n}}\right]^{\mathrm{m}}}
$$

em que:

$$
\begin{aligned}
& \mathrm{q}_{\mathrm{a}} \text { - umidade real da base em volume, } \mathrm{m}^{3} \mathrm{~m}^{-3} \\
& \mathrm{q}_{\mathrm{r}} \text { - umidade residual em volume, } \mathrm{m}^{3} \mathrm{~m}^{-3} \\
& \mathrm{q}_{\mathrm{s}} \text { - umidade de saturação em volume, } \mathrm{m}^{3} \mathrm{~m}^{-3} \\
& \mathrm{Y}_{\mathrm{m}} \text { - potencial da água no solo, cm.c. } \mathrm{a} \\
& \mathrm{a}, \mathrm{n} \text { e } \mathrm{m} \text { - parâmetros empíricos do modelo matemático. }
\end{aligned}
$$

O experimento foi irrigado por gotejamento em faixa contínua e instalados dois gotejadores por metro linear; dois dias antes do plantio, a área foi irrigada utilizando-se um sistema de irrigação por aspersão convencional, com o objetivo de uniformizar a umidade do solo. Após o plantio, a área passou a ser irrigada diariamente, utilizando-se o sistema de irrigação por gotejamento, até o início dos tratamentos.

Cada unidade experimental foi constituída de trinta plantas espaçadas 1,0 m entre plantas e 1,5 m entre linhas, com uma planta por cova e as plantas situadas nas laterais das parcelas serviram como bordadura. Cada parcela ocupou uma área de $45 \mathrm{~m}^{2}$, sendo que a área útil ocupou $18,0 \mathrm{~m}^{2}$. O delineamento 
experimental adotado foi o de blocos ao acaso, com oito tratamentos e quatro repetições cada um, totalizando trinta e duas parcelas.

O desenvolvimento da cultura foi dividido em três estádios fenológicos, de acordo com as observações de caráter morfológico: 1. estádio vegetativo - da emergência das plantas e estádio inicial estádio de floração; 2. estende-se do final do ciclo anterior até o início da formação dos frutos, desenvolvimento dos frutos e 3. do final da fase anterior até a colheita.

Os tratamentos foram definidos em função da época de aplicação do déficit hídrico e agrupados da seguinte forma: $\mathrm{T}_{1}$ - sem déficit hídrico, $\mathrm{T}_{2}$ - com déficit hídrico no estádio de desenvolvimento dos frutos, $\mathrm{T}_{3}$ - com déficit hídrico no estádio de floração, $\mathrm{T}_{4}$ - com déficit hídrico nos estádios de floração e desenvolvimento dos frutos, $\mathrm{T}_{5}$ - com déficit hídrico no estádio vegetativo, $\mathrm{T}_{6}$ - com déficit hídrico nos estádios vegetativo e desenvolvimento dos frutos, $\mathrm{T}_{7}$ - com déficit hídrico nos estádios vegetativo e floração e $\mathrm{T}_{8}$ - com déficit hídrico em todos os estádios.

Em cada parcela dos tratamentos foi instalada uma bateria com quatro tensiômetros, nas profundidades de 0,10, 0,30, 0,50 e $0,70 \mathrm{~m}$; as irrigações foram realizadas sempre que em pelo menos três repetições a umidade do solo fosse menor ou igual a $0,154 \mathrm{~m}^{3} \mathrm{~m}^{-3}$. As parcelas em déficit hídrico receberam $50 \%$ da média da lâmina aplicada nas parcelas do tratamento 1, quando este indicava o momento de se irrigar. A lâmina aplicada nos tratamentos que não se encontravam em déficit hídrico era a necessária para que a umidade do solo chegasse à capacidade de campo $\left(0,187 \mathrm{~m}^{3} \mathrm{~m}^{-3}\right)$.

Os tratos culturais, como capinas e controle de pragas e doenças, foram realizados no decorrer do ciclo da cultura, a medida em que se tornava necessário; as capinas foram efetuadas manualmente, com enxada, de acordo com a ocorrência de ervas daninhas e para as aplicações dos defensivos agrícolas utilizou-se um pulverizador costal, sendo que para o controle de pragas e doenças foram usados os defensivos Monocrotophos, Mancozeb e Confidor. A colheita das unidades experimentais foi realizada no dia 28 de dezembro de 1997, e em 1 e 5 de janeiro de 1998, quando as plantas apresentaram a maioria dos frutos maduros. Foram considerados maduros os frutos que apresentavam a gavinha seca e a colheita foi executada de maneira manual.

\section{Estimativa da evapotranspiração}

A evapotranspiração de referência $\left(\mathrm{ET}_{\mathrm{o}}\right)$ foi obtida através do método do tanque "Classe A". A equação utilizada para este método foi apresentada por Ometto (1981):

$$
\mathrm{ET}_{\mathrm{o}}=\mathrm{K}_{\text {pan }} . \mathrm{ECA}
$$

em que:

ECA - evaporação medida diariamente no tanque Classe A (mm)

$\mathrm{K}_{\text {pan }}$ - coeficiente de ajuste do tanque.

$\mathrm{O} \mathrm{K}_{\text {pan }}$ foi calculado através da equação proposta por Snyder (1992) que se segue abaixo:

$$
\mathrm{K}_{\text {pan }}=0,482+0,24 \ln (\mathrm{F})-0,00037 \mathrm{U}+0,0045 \mathrm{UR}
$$

sendo:

F - tamanho da área de bordadura, em m

$\mathrm{U}$ - velocidade do vento, em $\mathrm{km} \mathrm{dia}^{-1}$

UR - umidade relativa, em \%.

A evapotranspiração da cultura foi estimada mediante o método do balanço hídrico, que tem fundamento básico na lei da conservação das massas, e apresentada por Reichardt (1996) na forma simplificada, como:

$$
\mathrm{P}+\mathrm{I} \pm \mathrm{Q}_{\mathrm{Z}} \pm \mathrm{ET} \pm \mathrm{ES}= \pm \mathrm{Dh}
$$

em que $\mathrm{P}, \mathrm{I}, \mathrm{Q}_{\mathrm{Z}}, \mathrm{ET}$, ES e Dh representam, respectivamente, precipitação natural $(\mathrm{mm})$, irrigação $(\mathrm{mm})$, drenagem profunda ou ascensão capilar (mm), evapotranspiração da cultura (mm), escoamento superficial ( $\mathrm{mm}$ ) e variação do armazenamento (mm) da água do solo na camada de profundidade de 0 a $\mathrm{Z}$, para o intervalo de tempo considerado do balanço.

A Eq. 4 é comumente utilizada para efeito de balanço hídrico, porém para seu uso em solos cultivados é necessário considerar-se uma profundidade do volume de solo em estudo, que corresponde à profundidade efetiva do sistema radicular. No caso em questão, a profundidade considerada foi de $0,40 \mathrm{~m}$.

\section{Determinação dos componentes do balanço}

Os dados de precipitação pluvial utilizados no presente estudo foram obtidos em pluviômetro instalado no local do experimento.

As lâminas de irrigação foram definidas em função do potencial da água do solo nas profundidades de 0,10 e 0,30 m. As expressões para o cálculo das lâminas de irrigação são mostradas a seguir:

Camada (0 a 0,20 m)

$$
\mathrm{L}_{(0-0,20)}=\left(q_{\mathrm{cc} 0,10}-q_{\mathrm{a} 0,10}\right) \cdot 200
$$

Camada $(0,20$ a $0,40 \mathrm{~m})$

$$
L_{(0,20-0,40)}=\left(q_{c c 30}-q_{a 30}\right) \cdot 200
$$

em que:

$\mathrm{L}_{(0-0,20)}$ e $\mathrm{L}_{(0,20-0,40)}$ - lâmina de irrigação para as camadas de 0 a 0,20 e 0,20 a $0,40 \mathrm{~m}$ respectivamente $(\mathrm{mm})$;

$q_{c c 0,10}$ e $q_{c c 0,30}$ - umidade do solo correspondente às capacidades de campo, nas profundidades de 0,10 e 0,30 m, expressa em $\mathrm{m}^{3} \mathrm{~m}^{-3}$, respectivamente. Este parâmetro foi determinado em campo durante o ensaio, para estimar a condutividade hidráulica do solo. Os valores obtidos foram os seguintes: $q_{c c 0,10}=0,187 \mathrm{~m}^{3} \mathrm{~m}^{-3}$ e $q_{c c 0,30}=0,182 \mathrm{~m}^{3} \mathrm{~m}^{-3}$

$q_{a 0,10}$ e $q_{a 0,30}$ - umidade do solo no momento das irrigações, nas profundidades de 0,10 e $0,30 \mathrm{~m}$, respectivamente, em $\mathrm{m}^{3} \mathrm{~m}^{-3}$. 
Os componentes de drenagem profunda ou ascensão capilar da água do solo foram calculados para a profundidade de 0,40 m através da equação de Buckingham-Darcy escrita de forma simplificada por Reichardt (1985) como :

$$
\mathrm{Q}_{\mathrm{Z}}=-\mathrm{K}(\theta) \cdot \frac{\Delta \psi}{\Delta \mathrm{Z}}
$$

donde:

$\mathrm{K}(q)$ - condutividade hidráulica em relação ao valor da umidade do solo

$\mathrm{D} \mathrm{Y}_{\mathrm{T}} / \mathrm{DZ}$ - gradiente do potencial total da água no solo na profundidade $\mathrm{Z}$.

Aplicando-se a Eq. (6) para a profundidade de 0,40 m, obter-se-á a seguinte equação:

$$
\mathrm{Q}_{Z}=-\mathrm{K}(\theta)_{40}\left[\frac{\psi_{30}-\psi_{50}}{20}\right]_{40}
$$

em que:

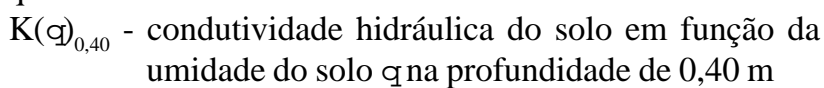

$\left[\frac{\psi_{30}-\psi_{50}}{20}\right]_{40}$

- gradiente de potencial total da água do solo na profundidade de $0,40 \mathrm{~m}$, sendo $\mathrm{Y}_{\mathrm{T} 30}$ o potencial total da água do solo a $0,30 \mathrm{~m} \mathrm{(cm} \mathrm{c} \mathrm{a)} \mathrm{e}{ }_{\mathrm{T} 50}$ o potencial total da água do solo a $0,50 \mathrm{~m} \mathrm{(cm} \mathrm{c} \mathrm{a).}$

Os valores de $\mathrm{K}(q)_{0,40}$ foram obtidos mediante um ensaio de campo realizado na área do experimento, numa parcela de $10 \mathrm{~m}^{2}$, com quatro baterias de tensiômetros de mercúrio nas profundidades de $0,10,0,30,0,50$ e $0,70 \mathrm{~m}$. O ensaio foi conduzido durante 40 dias, quando foi determinada a condutividade hidráulica a 0,40 m, conforme método de Hillel et al. (1972) modificado por Saunders (1978). A expressão obtida foi a seguinte:

$$
\mathrm{K}(\theta)_{40}=4,6708 \mathrm{e}^{16,569(\theta-0,415)}
$$

em que:

$\mathrm{K}(\mathrm{q})_{0,40}$ - condutividade hidráulica na profundidade de $0,40 \mathrm{~m}$

q- umidade média na camada de 0 a $0,40 \mathrm{~m}$.

A determinação da variação do armazenamento da água do solo na profundidade e no intervalo de tempo considerados, foi obtida mediante a expressão (Reichardt, 1985) :

$$
D h=\left(q_{f}-q_{i}\right) \cdot Z
$$

em que:

$\mathrm{q}_{\mathrm{f}}$ - umidade média do perfil até $0,40 \mathrm{~m}$, no tempo $\mathrm{T}_{\mathrm{f}}$, em $\mathrm{m}^{3} \mathrm{~m}^{-3}$

$\mathrm{q}_{\mathrm{i}}$ - umidade média do perfil até $0,40 \mathrm{~m}$, no tempo $\mathrm{T}_{i}$ em $\mathrm{m}^{3} \mathrm{~m}^{-3}$

$\mathrm{Z}$ - profundidade adotada para o balanço $(0,40 \mathrm{~m})$.

Nas condições em que o presente trabalho foi conduzido, o componente ES não foi considerado, visto que as unidades experimentais foram sistematizadas a nível e com bordas para evitar tal escoamento da água de irrigação.
Determinou-se a componente ET através da explicitação da Eq. (4) do balanço hídrico:

$$
\mathrm{ET}=\mathrm{P}+\mathrm{I} \pm \mathrm{Q}_{\mathrm{Z}}-\mathrm{Dh}
$$

Conforme as condições em que o ensaio foi desenvolvido, a evapotranspiração encontrada pode ser conceituada como evapotranspiração máxima $\left(E T_{m}\right)$, obtida através do tratamento 1, e real $\left(E T_{r}\right)$ obtida nos tratamentos em déficit hídrico nas diversas fases fenológicas da cultura.

\section{RESULTADOS E DISCUSSÃO}

O ciclo da cultura teve, desde a emergência até a colheita, duração de 68 dias, iniciando-se em 30 de outubro de 1997, data em que todas as covas semeadas apresentavam plântulas com duas folhas, terminando com a última colheita, em 5 de janeiro de 1998.

O desenvolvimento da cultura foi dividido em três estádios fenológicos, de acordo com as observações de caráter morfológico: Estádio vegetativo: caracterizado pela emergência de plantas e estádio inicial, estendeu-se do $12^{\circ}$ dia após a emergência até o início da floração (29 dias após a emergência) com duração total de 17 dias; estádio de floração: caracterizado pela emissão das flores e início da frutificação, prolongou-se do final da fase anterior até o início da formação dos frutos (47 dias após a emergência) com duração total de 19 dias; estádio de desenvolvimento dos frutos: do final da fase anterior até a colheita (71 dias após a emergência) com duração total de 24 dias. O balanço hídrico teve início em 7 de novembro de 1997 e foi aplicado nos três estádios fenológicos definidos.

A divisão do ciclo da cultura permitiu verificar-se o seu comportamento evapotranspiratório em cada estádio. Assim sendo verifica-se que nas parcelas do tratamento 1 que não sofreram restrição no suprimento de água, a $\mathrm{ET}_{\mathrm{m}}$ média no estádio vegetativo foi de $3,2 \mathrm{~mm} \mathrm{dia}^{-1}$. Para os estádios de floração e desenvolvimento dos frutos obtiveram-se 5,9 $\mathrm{mm} \mathrm{dia}^{-1}$ e 5,0 $\mathrm{mm} \mathrm{dia}^{-1}$, respectivamente.

Esses valores médios se assemelham ao valor encontrado por Ferreira (1990), utilizando o método do balanço hídrico em Pentecoste, CE, de 6,50 $\mathrm{mm}$ dia $^{-1}$ para a evapotranspiração máxima da melancia. Pode-se compará-los, também, aos obtidos com melão, por Miranda (1998) de 6,0 $\mathrm{mm} \mathrm{dia}^{-1}$ e Santos (1985) de $5,10 \mathrm{~mm} \mathrm{dia}^{-1}$; já nas parcelas do tratamento 8 , que receberam metade da lâmina média aplicada no tratamento 1 , durante os três estádios fenológicos, a $\mathrm{ET}_{\mathrm{r}}$ média foi de $2,18 \mathrm{~mm} \mathrm{dia}^{-1}$ no estádio vegetativo, de 4,06 $\mathrm{mm} \mathrm{dia}^{-1}$ no estádio de floração e de 2,48 $\mathrm{mm} \mathrm{dia}^{-1}$ no estádio de desenvolvimento dos frutos e corresponderam a $67,31 \%, 68,80 \%$ e $49,90 \%$ da evapotranspiração máxima obtida no tratamento 1 , nos respectivos estádios fenológicos.

A evolução da evapotranspiração nos tratamentos é apresentada na Figura 1, na qual se mostra o contraste entre o tratamento $1\left(\mathrm{ET}_{\mathrm{m}}\right)$ sem déficit hídrico, o tratamento $8\left(\mathrm{ET}_{\mathrm{r}}\right) \mathrm{com}$ déficit hídrico em todo o ciclo e a evapotranspiração de referência $\left(\mathrm{ET}_{\mathrm{o}}\right)$.

Os valores de $\mathrm{ET}_{\mathrm{r}}, \mathrm{ET}_{\mathrm{m}}, \mathrm{y}_{\mathrm{r}}, \mathrm{y}_{\mathrm{m}},\left(1-\mathrm{ET}_{\mathrm{r}} / \mathrm{ET}_{\mathrm{m}}\right),\left(1-\mathrm{y}_{\mathrm{r}} / \mathrm{y}_{\mathrm{m}}\right)$ e os coeficientes de sensibilidade $\mathrm{k}_{\mathrm{y}}$ médios calculados para cada tratamento sob déficit hídrico nos estádios fenológicos da melancia, são apresentados na Tabela 1 . 
Tabela 1: Valores médios de $\left[1-\left(\mathrm{ET}_{\mathrm{r}} / \mathrm{ET}_{\mathrm{m}}\right)\right]$ e $\left[1-\left(\mathrm{y}_{\mathrm{r}} / \mathrm{y}_{\mathrm{m}}\right)\right]$ e do fator de sensibilidade $\mathrm{k}_{\mathrm{y}}$ da melancia, cultivar Crimson Sweet, nos tratamentos sob déficit hídrico

\begin{tabular}{crrccccccc}
\hline Tratamento & \multicolumn{1}{c}{$E T_{r}$} & \multicolumn{1}{c}{$E T_{m}$} & $E T_{r} / E T_{m}$ & $\left(1-E T_{r} / E T_{m}\right)$ & $y_{r}$ & $y_{m}$ & $y_{r} / y_{m}$ & $\left(1-y_{r} / y_{m}\right)$ & $k_{y}$ \\
\hline $\mathrm{T}_{2}$ & 87,06 & 119,30 & 0,7298 & 0,2702 & $53.230,51$ & $63.385,93$ & 0,8398 & 0,1602 & 0,59 \\
$\mathrm{~T}_{3}$ & 92,02 & 112,22 & 0,8200 & 0,1800 & $51.734,48$ & $63.385,93$ & 0,8162 & 0,1838 & 1,02 \\
$\mathrm{~T}_{4}$ & 177,84 & 231,52 & 0,7681 & 0,2319 & $48.287,26$ & $63.385,93$ & 0,7618 & 0,2382 & 1,03 \\
$\mathrm{~T}_{5}$ & 27,24 & 55,07 & 0,4946 & 0,5054 & $59.632,25$ & $63.385,93$ & 0,9408 & 0,0592 & 0,12 \\
$\mathrm{~T}_{6}$ & 110,68 & 174,37 & 0,6347 & 0,3653 & $47.466,38$ & $63.385,93$ & 0,7488 & 0,2512 & 0,69 \\
$\mathrm{~T}_{7}$ & 117,71 & 167,29 & 0,7036 & 0,2964 & $47.901,98$ & $63.385,93$ & 0,7557 & 0,2443 & 0,82 \\
$\mathrm{~T}_{8}$ & 173,81 & 286,59 & 0,6065 & 0,3935 & $36.854,28$ & $63.385,93$ & 0,5814 & 0,4186 & 1,06 \\
\hline
\end{tabular}

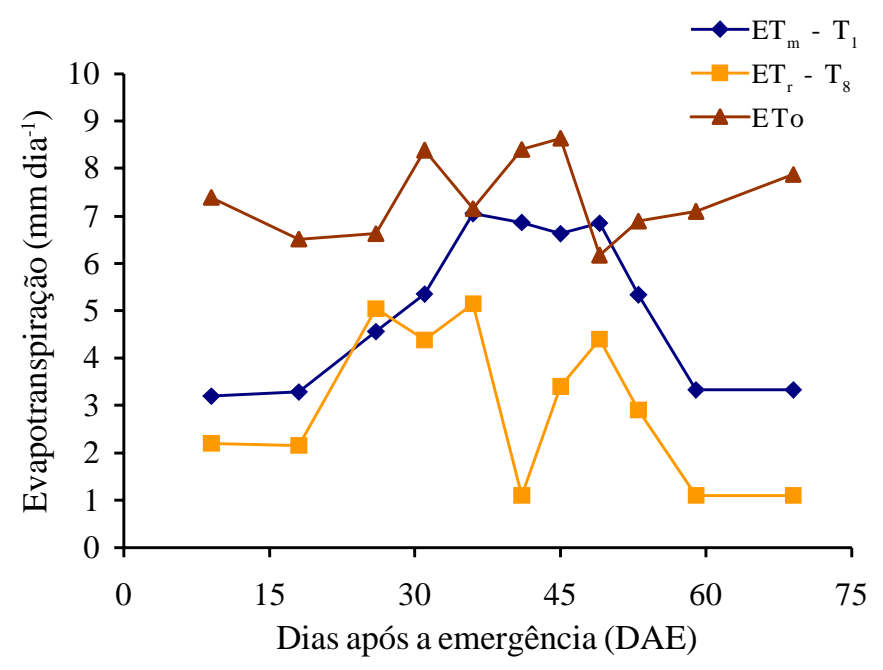

Figura 1. Evolução da evapotranspiração de referência em Canindé, CE, e das evapotranspirações real e máxima da melancia

Em condições de suprimento de água limitado, a cultura sofrerá maior perda de rendimento quanto maior for o valor de $\mathrm{k}_{\mathrm{y}}$ (Doorenbos \& Kassam, 1994). Analisando-se os valores de $\mathrm{k}_{\mathrm{y}}$ dos tratamentos 2, 3 e 5, que foram submetidos ao déficit hídrico em apenas um estádio fenológico, observa-se que a redução relativa da melancia foi mais acentuada no estádio de floração (tratamento 3 ) com valor de $\mathrm{k}_{\mathrm{y}}=1,02$. O déficit hídrico nos tratamentos 2 e 5 nos estádios de desenvolvimento dos frutos e vegetativo, respectivamente, teve pouco efeito sobre o rendimento da cultura, registrando valores de $\mathrm{k}_{\mathrm{y}}=0,15 \mathrm{na}$ fase vegetativa e de $\mathrm{k}_{\mathrm{y}}=0,57$ no desenvolvimento dos frutos.

Comparando-se esses valores com os obtidos por Doorenbos \& Kassam (1994) ao longo dos estádios fenológicos, observam-se valores diferentes nos três estádios fenológicos; assim, na floração o valor do $\mathrm{k}_{\mathrm{y}}=0,80$ para Doorenbos \& Kassam, contra $\mathrm{k}_{\mathrm{y}}=1,02$ para o presente estudo; na fase vegetativa $\mathrm{k}_{\mathrm{y}}=0,70$ e 0,45 para Doorenbos \& Kassam (dividem a fase em vegetativa inicial e final) contra $\mathrm{k}_{\mathrm{y}}=0,15 \mathrm{e}$ desenvolvimento dos frutos $\mathrm{k}_{\mathrm{y}}=0,3$ e 0,8 para Doorenbos e Kassam (dividem a fase em formação da colheita e maturação) contra $\mathrm{k}_{\mathrm{y}}=0,57$ para o presente estudo. Se, porém, nesse estádio for considerada a média dos valores obtidos na formação da colheita $\left(\mathrm{k}_{\mathrm{y}}=0,8\right)$ e maturação $\left(\mathrm{k}_{\mathrm{y}}=0,3\right)$ por Doorenbos e Kassam (1994) ter-se-á $\mathrm{k}_{\mathrm{y}}=0,55$ contra $\mathrm{k}_{\mathrm{y}}=0,57$, para o presente estudo.
A diferença de valores de $\mathrm{k}_{\mathrm{y}}$ encontrada nos estádios vegetativo e de floração, em relação ao valor apresentado pela FAO, pode ter sido influenciada pelas condições ambientais, pela cultivar utilizada e sua adaptação a esse ambiente e, ainda, pela divisão das fases fenológicas da cultura. Outra explicação para as diferenças dos resultados obtidos é que, segundo Doorenbos e Kassam (1994) uma avaliação dos dados experimentais de campo indica certa dispersão nos valores de $\mathrm{k}_{\mathrm{y}}$, que se deve à imperfeição experimental e às variações de clima, nível de evapotranspiração e solo; além do mais, deve-se ressaltar, conforme os próprios autores citados acima, que não se dispõe de valores-padrão de referências para comparação, mas apenas se supõe que a confiabilidade dos valores de $\mathrm{k}_{\mathrm{y}}$ apresentados pela FAO, é semelhante à procedente da análise dos resultados experimentais do campo.

Dos tratamentos com estresse hídrico em mais de um estádio fenológico, o que apresentou o maior coeficiente de sensibilidade $\mathrm{k}_{\mathrm{y}}$ foi o tratamento 8 com $\mathrm{k}_{\mathrm{y}}=1,06$, o qual foi submetido a déficit hídrico durante o ciclo da cultura. Nos tratamentos com estresse hídrico em duas fases, o maior valor de $\mathrm{k}_{\mathrm{y}}$ ocorreu no tratamento $4\left(\mathrm{k}_{\mathrm{y}}=1,03\right)$ com déficit hídrico nos estádios de floração e desenvolvimento dos frutos; logo em seguida, tem-se o tratamento 7 com $\mathrm{k}_{\mathrm{y}}=0,82$, que sofreu déficit hídrico nos estádios vegetativo e de floração. Observa-se que a fase que apresenta maior sensibilidade à restrição no suprimento de água é a de floração, visto que os tratamentos 3 e 4 apresentaram valores de $\mathrm{k}_{\mathrm{y}}$ superiores a 1 .

A produtividade de $63.375,93 \mathrm{~kg} \mathrm{ha}^{-1}$ obtida no tratamento 1, aproxima-se dos resultados observados por Andrade Jr. et al. (1997) e Rudich et al. (1978) que obtiveram produtividade máxima de 65,4 e $54,4 \mathrm{t} \mathrm{ha}^{-1}$, respectivamente.

\section{CONCLUSÕES}

Com os resultados obtidos e para as condições em que foi desenvolvido este experimento, pode-se concluir que:

1. O balanço hídrico em volume de solo controlado em campo, apresentou-se confiável na determinação do consumo de água pela cultura.

2. Os valores médios da evapotranspiração máxima da cultura $\left(\mathrm{ET}_{\mathrm{m}}\right)$ foram 3,2 $\mathrm{mm} \mathrm{dia}^{-1}$ no estádio vegetativo, $5,9 \mathrm{~mm} \mathrm{dia}^{-1}$ no estádio de floração e $5,0 \mathrm{~mm} \mathrm{dia}^{-1}$ no estádio de desenvolvimento dos frutos, perfazendo a média de $4,8 \mathrm{~mm}$ dia- $^{-1}$ para os três estádios em conjunto.

3. A fase de maior demanda evapotranspiratória foi a de floração, chegando a $7,1 \mathrm{~mm} \mathrm{dia}^{-1}$ no $36^{\circ} \mathrm{DAE}$ superior, portanto, à ET . 
4. A deficiência hídrica em uma fase fenológica provocou maior redução na produtividade, quando aplicada durante o estádio de floração.

5. Os valores dos coeficientes de sensibilidade $\mathrm{k}_{\mathrm{y}}$ encontrados para os tratamentos 3,4 e 8 foram superiores a 1 , indicando alta sensibilidade ao déficit hídrico.

6. A sensibilidade ao déficit hídrico $\left(\mathrm{k}_{\mathrm{y}}\right)$ em cada estádio variou na seguinte ordem decrescente: floração, desenvolvimento dos frutos e vegetativo, para déficit aplicado em apenas um estádio fenológico. Para as condições de déficit em dois estádios, o maior valor encontrado foi no tratamento 4 (déficit hídrico nos estádios de floração e desenvolvimento dos frutos).

7. A restrição da irrigação no estádio vegetativo pode ser aplicada à cultura, pois não resultou em queda significativa de produtividade.

\section{REFERÊNCIAS BIBLIOGRÁFICAS}

ANDRADE JR., A.A.; RODRIGUES, B.H.N.; ATHAYDE SOBRINHO, C.; MELO, F.B; BASTOS, E.A; CARDOSO, M.J.; RIBEIRO, V.Q. Produtividade e qualidade dos frutos de melancia, e função de diferentes níveis de irrigação. Horticultura Brasileira, Brasília, v.15, n.1, p.43-46, 1997.

ANUÁRIO ESTATÍSTICO DO BRASIL - 1996. IBGE, Rio de Janeiro, v.3, p.46.

ANUÁRIO ESTATÍSTICO DO CEARÁ - 1995. IPLANCE, Fortaleza, p.348-349.

DOORENBOS, J.; KASSAM, A.H. Efeito da água no rendimento das culturas. Campina Grande: UFPB, 1994. 306p. Estudos FAO: Irrigação e Drenagem, 33

DOURADO NETO, D.; LIER, Q.J.V.; BOTREL, T.A. et al. Curva de retenção de água do solo: Algoritmo em Quickbasic para estimativa dos parâmetros empíricos do modelo de Genuchten. Piracicaba: ESALQ, 1990. 32p. Manual de Instruções ao Usuário
FERREIRA, L.N.M. Determinação da evapotranspiração atual e potencial da cultura da melancia. Fortaleza: Universidade Federal do Ceará. 1990. 66p. Dissertação Mestrado

HILLEL, D.; KRENTOS, V.D.; STYLIANOV, Y. Procedure and test of an internal drainage method for measuring soil hydraulic characteristics in situ. Soil Science, Baltimor, v.114, p. 395-400, 1972.

MIRANDA, E.P. Coeficiente de cultivo do melão(Cucumis melo, L.) determinado pelo balanço hídrico e graus-dia de desenvolvimento. Fortaleza: Universidade Federal do Ceará. 1998. 61p. Dissertação Mestrado

OMETTO, J. C. Bioclimatologia vegetal. São Paulo, Agronômica Ceres, 1981. 425p.

REICHARDT, K. Processo de transferência no sistema solo-água-atmosfera. 4.ed. Campinas: Fundação Cargill, 1985. 466p.

REICHARDT, K. Á água em sistemas agrícolas. São Paulo: Manole, 1990. 188p.

REICHARDT, K. Dinâmica da matéria e da energia em ecossistemas. Piracicaba: Escola Superior de Agricultura "Luiz de Queiroz", USP. 1996, 513p.

SANTOS, G.A.S. Uso consuntivo da cultura do melão (Cucumis melo, L.). Fortaleza: Universidade Federal do Ceará. 1985. 71p. Dissertação Mestrado

SAUNDERS, L.C.U. Métodos de determinação e variabilidade espacial da condutividade hidráulica sob condições de campo. Piracicaba: ESALQ. 1978. 71p. Tese Doutorado

SNYDER, R.L. Equation for evaporation pan to evapotranspiration conversions. Journal of Irrigation and Drainage, England, v.118, p. 977-980, 1992

VIANELLO, R. L.; ALVES, A. R. Meteorologia básica e aplicações. Viçosa: UFV, Imprensa Universitária,1991. 449p. ils. 